\title{
COMPARACIÓN DE OPCIONES PARA INTELIGENCIA DE NEGOCIOS EN LOS PRINCIPALES SISTEMAS GESTORES DE BASES DE DATOS DEL MERCADO
}

ISSN 2219-6722

ISSNE 2222-2707

\author{
MARVIN AGUILAR ROMERO \\ Universidad Nacional Autónoma de Honduras (UNAH) \\ Correo electrónico: marvin.aguilar@unah.edu.hn \\ JOSÉ LUIS RODRÍGUEZ GARCÍA \\ Universidad Nacional Autónoma de Honduras (UNAH)
}

\section{RESUMEN}

El tener información en tiempo y forma es una de las mayores exigencias de hoy en día en todos los ámbitos para la toma de decisiones esto lleva a las instituciones a tener que invertir en sistemas de información robustos para inteligencia de negocios los cuales a su vez requieren de diversas tecnologías dispuestas de manera eficiente para el procesamiento de la información, debido a las múltiples herramientas existentes en las organizaciones lograr unificar las estructuras de datos es tarea compleja y costosa, sin embargo el uso de un sistema gestor de bases de datos que preste la mayoría de los servicios necesarios para un modelo de inteligencia de negocios supone una disminución en el uso de los recursos.

En la medida que el tiempo pasa se denota la influencia de la información al momento de tomar una decisión que afecte tanto el modelo de negocio así como la organización e interoperabilidad de una institución, así como también se denota cada vez más el acuñamiento y cimentación de la inteligencia de negocios dentro del diario vivir de las instituciones

Palabras clave: Sistemas Gestores de Bases de Datos, Minería de Datos, Inteligencia de Negocios. 


\title{
COMPARISON OF OPTIONS FOR INTELLIGENCE BUSINESS IN MAJOR SYSTEMS MANAGERS MARKET DATABASES
}

ISSN 2219-6722

ISSNE 2222-2707

MARVIN AGUILAR ROMERO

Universidad Nacional Autónoma de Honduras (UNAH)

Correo electrónico: marvin.aguilar@unah.edu.hn

JOSÉ RODRÍGUEZ GARCÍA

Universidad Nacional Autónoma de Honduras (UNAH)

\begin{abstract}
Having information in a timely manner is one of the major demands of today in all areas of decision making that leads to the institutions have to invest in systems robust information for business intelligence which in turn require various technologies arranged efficiently for information processing manner, due to the many tools that exist in organizations to achieve unified data structures is complex and expensive task, however the use of a management system database to provide the most of the necessary services for business intelligence model is a decrease in the use of resources.
\end{abstract}

To the extent that time passes the influence of information when making a decision affecting both the business model and the organization and interoperability of an institution is denoted as well as denoted increasingly wedging and foundation business intelligence into the daily life of institutions

Keywords: Data Base System Management, Data Mining, Bussiness Intelligence. 


\section{INTRODUCCIÓN}

El presente trabajo expone las diferencias existentes entre los Sistemas Gestores de Bases de Datos más populares tomando como referencia su aporte a la inteligencia de negocio; Se inicia exponiendo la terminología de inteligencia de negocio luego se trata la importancia de contar un buen SGBD cuando se realiza ésta operación.

Se realiza una comparativa de los Sistemas Gestores de Bases de Datos más populares desde un punto de vista para prestación de servicios de inteligencia de negocios sin necesidad de emplear módulos o herramientas adicionales.

Se muestra una comparativa tanto de requerimientos de software y hardware hasta costos por licenciamiento de los SGBD esto para contextualizar los costos y necesidades de cada uno de estos, así como contrastar la inversión en aprendizaje para el manejo certificado de cada una de estas herramientas.

Finalmente, y a partir de la evidencia se comienza la evaluación de los SGBD para determinar que SGBD es más conveniente utilizar cuando se desea realizar Inteligencia de Negocios.

\section{MARCO REFERENCIAL}

\subsection{INTELIGENCIA DE NEGOCIO}

Éste término usado por primera vez en 1958 por Hans Peter Luhn (Luhn, 1958) puede resumirse sencillamente como la adquisición (recopilación) y uso de conocimiento basado en hechos con la finalidad de mejorar la estrategia de negocio y las ventajas tácticas en el mercado (Oramas, 2009). Concepto que denota una práctica que puede ser útil para todas las organizaciones sin importar su rubro y que, además, precisa el uso de las tecnologías de información y las telecomunicaciones, sin embargo, esto último no fue siempre así.

Por algún tiempo autores y profesionales en dirección empresarial consideraban que era necesario la contratación de personal dedicado de manera exclusiva a la inteligencia de negocios (Macías Mesa \& Artola Pimentel, 2009) dada la naturaleza de la misma que requiere reunir información de fuentes primarias y secundarias sobre bases de conocimiento, actualización de la información para posterior análisis, generar y establecer posibles elementos de cambio y difundir la acción oportuna para que a las personas que corresponde la toma de decisiones puedan elegir opciones que permitan una verdadera ventaja competitiva pero hoy en día, debido a las bondades de la técnica y a la tecnología disponible, muchas empresas de desarrollo de software así como corporaciones insignia de las TICs desarrollaron sistemas de información orientados al proceso de Inteligencia de Negocios y en la actualidad para grandes corporaciones el uso de estas herramientas es un activo más obligatorio en sus libros.

La eficacia de estas nuevas soluciones se puede valorar a partir de 5 indicadores vitales ${ }^{1}$ en el proceso de facilitar información para la toma de decisiones:

1 Vistos más desde el punto de vista gerencial que del informático. 
1. Rapidez: Facilitar a usuario la información procesada en la menor cantidad de tiempo posible.

2. Fiabilidad: Relativo a la confianza que puede tenerse sobre la información presentada.

3. Nivel de abstracción: capacidad de dar respuestas a preguntas complejas en base al procesamiento de unidades más pequeñas de información.

4. Navegación en profundidad: La versatilidad con la que se cambia de perspectiva desde lo general a lo específico.

5. Presentación de la información: Interfaz de calidad que permita las operaciones e interpretación de manera natural e intuitiva.

\subsection{ARQUITECTURA BÁSICA PARA IMPLEMENTACIÓN DE UN MODELO DE INTELIGEN- CIA DE NEGOCIOS}

Citando a Araníbar "todas las decisiones que se ejecutan en una empresa deberían provenir de las propias operaciones empresariales y de fenómenos que se producen en el entorno organizacional. Expresado todo en términos de información" (Araníbar S., 2003) por ello es necesario contar con un método de calidad para la gestión de las fuentes y tratamiento de su transformación (pasar de datos a información y luego conocimiento) hasta que se convierta en el insumo que alimente los sistemas de inteligencia de negocios.

Para poder tomar una decisión los sistemas de apoyo deben facilitar conocimiento en forma de hechos, información en la que se garantiza su fiabilidad desde antes incluso de ser transformada, y es que debido a la diversidad de las fuentes de datos es compleja la unificación de los mismos, un error en éste nivel podría arrojar como salida información que pareciere correcta porque es válida en su proceso, pero no legítima en su base lo que ocasionaría la toma de una decisión errónea.

Esto anterior da pie a las bases que deben regir la mecánica interna de los sistemas de Inteligencia de Negocio:

1. Administración de repositorios de datos (Datawarehouse, data mart, big data, entre otros): Espacio donde se realiza la extracción de la información a partir de las fuentes y se centraliza en una estructura dispuesta para las fases posteriores, acá los datos se validan y se limpian para no filtrar errores en el proceso.

2. Minería de datos (Data Mining): Conjunto de procesos que permiten extraer hechos a partir de las fuentes en los repositorios cuidando la integridad referencial de la información:

3. Procesamiento Analítico en Línea (OLAP - Online Analytical Processing): En ésta etapa se conforman "cubos" que pueden responder a uno de los tres esquemas existentes para OLAP:

MOLAP: OLAP Multidimensional hace uso de una base de datos optimizada para el almacenamiento de la información especializada.

ROLAP: OLAP Relacional En este tipo de implementación los hechos se construyen a 
partir de unidades de información no multidimensionales.

HOLAP: OLAP Híbrido: Posee elementos lineales y elementos que requieren almacenamientos especiales.

Resumidos en un lenguaje no tan técnico como "preparación de datos, modelado y evaluación” (Llombart, 2004).

La figura 1 resume el proceso y las interacciones entre estos y otros elementos que componen un sistema completo de ingeniería del negocio.

Figura No. 1. Elementos que constituyen un modelo de Inteligencia de Negocios

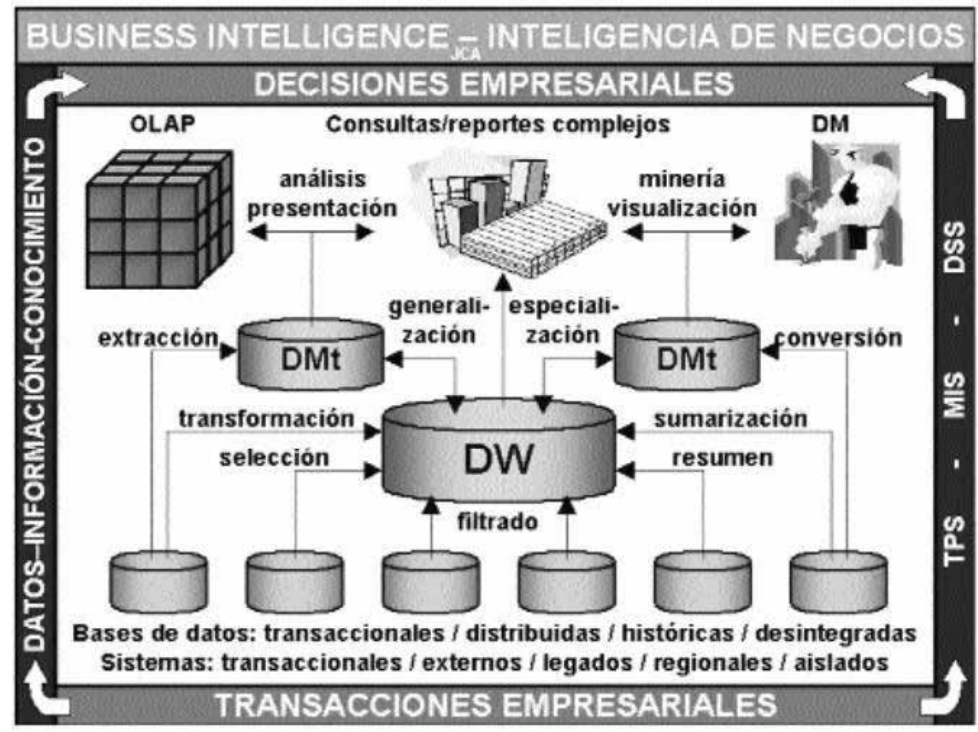

(Fuente: (Araníbar S., 2003)

\subsection{SISTEMA GESTOR DE BASES DE DATOS}

Un sistema gestor de bases de datos (SGBD) pueden verse como una capa intermedia que integra el DML y el DDL para facilitar la gestión de tuplas y consultas generalmente basadas en un estándar de un lenguaje para ejecución de consultas (SQL). Un SGBD debe facilitar las siguientes tareas (Zambrano Ramírez, 2008):

1. Definición y creación de las bases de datos.

2. Manipulación de los datos realizando consultas, inserciones y actualizaciones.

3. Acceso controlado a los datos mediante mecanismos de seguridad de acceso a los usuarios.

4. Mantener la integridad de los datos.

5. Controlar la concurrencia a la base de datos.

6. Mecanismos de copias de respaldo y recuperación para restablecer la información en caso de fallos de sistema. 
A la fecha son muchos los SGBD que se han desarrollados algunos como FoxPro han desaparecido del mercado y otros como ORACLE se han convertido en herramientas aterradoramente potentes con funcionalidades que van más allá de las requeridas por un SGBD. Esto es tanto así que la mayoría de los SGBD modernos cuentan con facilidad de conectarse a la una gran variedad de lenguajes de programación además de presentar interfaces gráficas llamativas y funcionales que ahorran muchas horas en la construcción de instrucciones por líneas de comando.

\subsection{SGBD DISPUESTO PARA INTELIGENCIA DE NEGOCIOS}

Tal como hemos mencionado anteriormente la inteligencia de negocios requiere de un componente base que trata de la recolección y transformación de los datos en las fuentes a un área centralizada para depositarse y de ahí ser analizados, el proceso de Recolección, Transformación y Carga conocido como ETL por sus siglas en inglés es una funcionalidad de creciente obligatoriedad debido a la gran cantidad de información y de diversidad de fuentes en el mercado.

La recopilación de datos a partir de transacciones se desarrolla casi de manera exclusiva en algún SGBD que, dependiendo de diversos factores, puede o no ser un sistema de software libre o propietario, pero hay que recordar que el diseño de bases de datos transaccionales no se pensó orientado a la extracción de la información (Llombart,

2004) es por ello que se requiere del ahora conocido ETL para lograr "cargar" en un nuevo repositorio los datos depurados, esta novedad puede ser un DataWarehouse o un Data mart donde "Se llama datawarehouse al almacén de datos que reúne la información histórica generada por todos los distintos departamentos de una organización, orientada a consultas complejas y de alto rendimiento. Un datawarehouse tiene una orientación corporativa que pretende conseguir que cualquier departamento pueda acceder a la información de cualquiera de los otros mediante un único medio, así como obligar a que los mismos términos tengan el mismo significado para todos. Un datamart es un almacén de datos históricos relativos a un departamento de una organización, así que puede ser simplemente una copia de parte de un datawarehouse para uso departamental. Tanto el datawarehouse como el datamart son sistemas orientados a la consulta, en los que se producen procesos en lote (batch) de carga de datos (altas) con una frecuencia baja y conocida." (Gascón \& Castellar-Busó, 2001).

Comenzaremos entonces con el proceso que deben poseer los SGBD para poder brindar soporte a sistemas de BI.

1. Preparación de los datos: El SGBD debe poseer la facilidad en la carga y exportación de la información, además facilitar conectores (OBDC) a la mayor cantidad de lenguajes de programación posible, así como de herramientas para migración de datos.

2. Gestión del almacén de datos: El SGBD debe ser capaz de manejar grandes volúmenes de información en la menor cantidad de tiempo posible además de permitir opciones nativas propias de algoritmos de minería de datos y de tratamiento de datos.

3. Soporte para cubos: Estructuras donde se centraliza información obtenida del almacén de datos que permite obtener hechos y modelar los mismos a través de diversas dimensiones que lo componen obteniendo así KPIS. 
4. Procesamiento de CUBOS: Realizar las operaciones OLAP con la mayor brevedad de tiempo posible, así como facilitar una interfaz amigable.

La legitimidad se ha descrito como "una percepción o suposición generalizada de que las acciones de una entidad son deseables, propia o apropiada dentro de un sistema socialmente estructurado de normas, valores, creencias y definiciones" (Suchman, 1995).

\section{METODOLOGÍA}

Para el desarrollo del presente estudió se procedió con el análisis comparativo de los SGBD más usados en la actualidad para luego comparar si los mismos satisfacían (y en qué medida) los requerimientos para considerarse herramientas sugeridas para el proceso de inteligencia de negocios

Entre los SGBD existentes tomaremos 6 para evaluarlos y compararlos conforme a su utilidad para inteligencia de negocios, los SGBD seleccionados son:

1. ORACLE (ORACLE, 2013).

2. SQL Server (Microsoft, 2013).

3. DB2 (IBM, 2013).

4. MySQL (ORACLE - MySQL, 2013).

5. PostgreSQL (PostgreSQL, 2013).

6. SYBASE (SYBASE, 2013).

\section{ANÁLISIS DE DATOS Y RESULTADOS}

\subsection{Opciones Nativas}

Hemos de considerar como opciones nativas aquellas funciones propias de un SGBD que manipulan de manera directa mediante el DML y el DDL las tuplas contenidas, un ejemplo de esto es la función para insertar un nuevo registro, la inserción se realiza de manera directa sobre el SGBD y aunque puede usarse un componente adicional para realizar esta labor (mediante algún módulo de php por ejemplo) el SGBD no requiere agente exterior para realizarla.

En el caso de nuestros 6 SGBD todos, aunque de diferente forma, permiten las funciones básicas para ETL, La tabla 1 muestra resume esta información:

1. Extracción: Todos los SGBD pueden exportar mediante un agregado a la sentencia SELECT la información en los registros de sus tablas.

2. Transformación: Cada SGBD facilita el cambio de tipo de cada relación en las tablas de los esquemas.

3. Carga: El mecanismo de INSERT sobrecargado o no está disponible para los 6 SGBD. 
TABLA No. 1. ETL nativo para SGBD

\begin{tabular}{|l|c|c|c|}
\hline SGBD & \multicolumn{1}{|c|}{ Extracción } & Transformación & Carga \\
\hline ORACLE & Sí & Sí & Sí \\
\hline $\begin{array}{l}\text { SQL } \\
\text { SERVER }\end{array}$ & Sí & Sí & Sí \\
\hline DB2 & Sí & Sí & Sí \\
\hline MySQL & Sí & Sí & Sí \\
\hline PosgreSQL & Sí & Sí & Sí \\
\hline SYBASE & Sí & Sí & Sí \\
\hline
\end{tabular}

Fuente: Elaboración propia a partir de manuales de referencia de los SGBD.

Sin embargo, lo que nos interesa en mayor medida es el grado de integración que poseen estos SGBD para inteligencia del negocio, para ello hemos de evaluarlos con las dos operaciones propias para el tratamiento de DW el Roll-UP y el Drill-DOWN. La tabla 2 muestra el resumen de ésta comparativa. ROLL-UP y DRILL-DOWN son técnicas que permiten la navegación a través de distintos niveles de detalle de una jerarquía de datos desde los más generales hasta los más específicos por ejemplo se pueden sumarizar las ventas por país y luego querer ver dentro del país que ciudades aportaron un mayor porcentaje sobre las ventas registradas (Alvarez, Gorza, \& Lesie, 2011).

TABLA No. 2. Funciones DW nativas por SGBD

\begin{tabular}{|c|c|c|c|c|c|c|}
\hline Funcionalidad & ORACLE & $\begin{array}{l}\text { SQL } \\
\text { SERVER }\end{array}$ & $\overline{\text { DB2 }}$ & MySQL & PosgreSQL & $\overline{\text { SyBASE }}$ \\
\hline ROLL-UP & Sí & Sí & Sí & Sí & Sí & Sí \\
\hline $\begin{array}{l}\text { DRILL- } \\
\text { DOWN }\end{array}$ & Sí & Sí & Sí & Sí & Sí & Sí \\
\hline
\end{tabular}

Fuente: Elaboración propia a partir de manuales de referencia de los SGBD.

Como puede apreciarse en éstas comparaciones básicas todos los SGBD satisfacen los requerimientos solicitados. Es entonces hora de comenzar la comparación para funciones OLAP pero para poder realizar la comparación es necesario identificar entre versiones de los SGBD ya que la inclusión o no del componente depende de la versión para efectos del análisis, de momento solo hemos de mencionar si de forma nativa ya sea mediante un módulo pagado o no los SGBD satisfacen los requerimientos para tratamientos de CUBOS (OLAP) la tabla 3 presenta los resultados. 
TABLA No. 3. Funciones OLAP nativas por SGBD

\begin{tabular}{|l|c|c|c|c|c|c|}
\hline Funcionalidad & ORACLE & SQL SERVER & DB2 & MySQL & PosgreSQL & SyBASE \\
\hline SLICE \& DICE & Sí & Sí & Sí & Sí & No & Sí \\
\hline PIVOT & Sí & Sí & Sí & Sí & No & Sí \\
\hline $\begin{array}{l}\text { DRILL- } \\
\text { THROUGH }\end{array}$ & Sí & Sí & Sí & Sí & No & Sí \\
\hline DRILL-ACROSS & Sí & Sí & Sí & Sí & No & Sí \\
\hline
\end{tabular}

Fuente: Elaboración propia a partir de manuales de referencia de los SGBD.

En la tabla 4 hacemos finalmente la comparación entre SGBD para determinar si pueden o no de manera nativa dar soporte a la inteligencia de negocios, la gráfica 1 ilustra el resumen de lo que se concluye a partir de la información en la tabla 4.

TABLA No. 4. Total, de funciones de necesarias para inteligencia de negocio por SGBD

\begin{tabular}{|c|c|c|c|c|c|c|c|}
\hline & Funcionalidad & ORACLE & $\begin{array}{l}\text { SQL } \\
\text { SERVER }\end{array}$ & DB2 & MySQL & PosgreSQL & SyBASE \\
\hline \multirow[t]{4}{*}{ 密 } & $\begin{array}{ll}\text { SLICE } & \& \\
\text { DICE } & \end{array}$ & 1 & 1 & 1 & 1 & 0 & 1 \\
\hline & PIVOT & 1 & 1 & 1 & 1 & 0 & 1 \\
\hline & $\begin{array}{l}\text { DRILL- } \\
\text { THROUGH }\end{array}$ & 1 & 1 & 1 & 1 & 0 & 1 \\
\hline & $\begin{array}{l}\text { DRILL- } \\
\text { ACROSS }\end{array}$ & 1 & 1 & 1 & 1 & 0 & 1 \\
\hline \multirow{2}{*}{ 党 } & ROLL-UP & 1 & 1 & 1 & 1 & 1 & 1 \\
\hline & $\begin{array}{l}\text { DRILL- } \\
\text { DOWN }\end{array}$ & 1 & 1 & 1 & 1 & 1 & 1 \\
\hline$\stackrel{\overrightarrow{\mid}}{\vec{\varphi}}$ & ETL & 1 & 1 & 1 & 1 & 1 & 1 \\
\hline & TOTAL: & $100 \%$ & $100 \%$ & $100 \%$ & $100 \%$ & $43 \%$ & $100 \%$ \\
\hline
\end{tabular}


Figura No. 2. Funciones para BI por SGBD

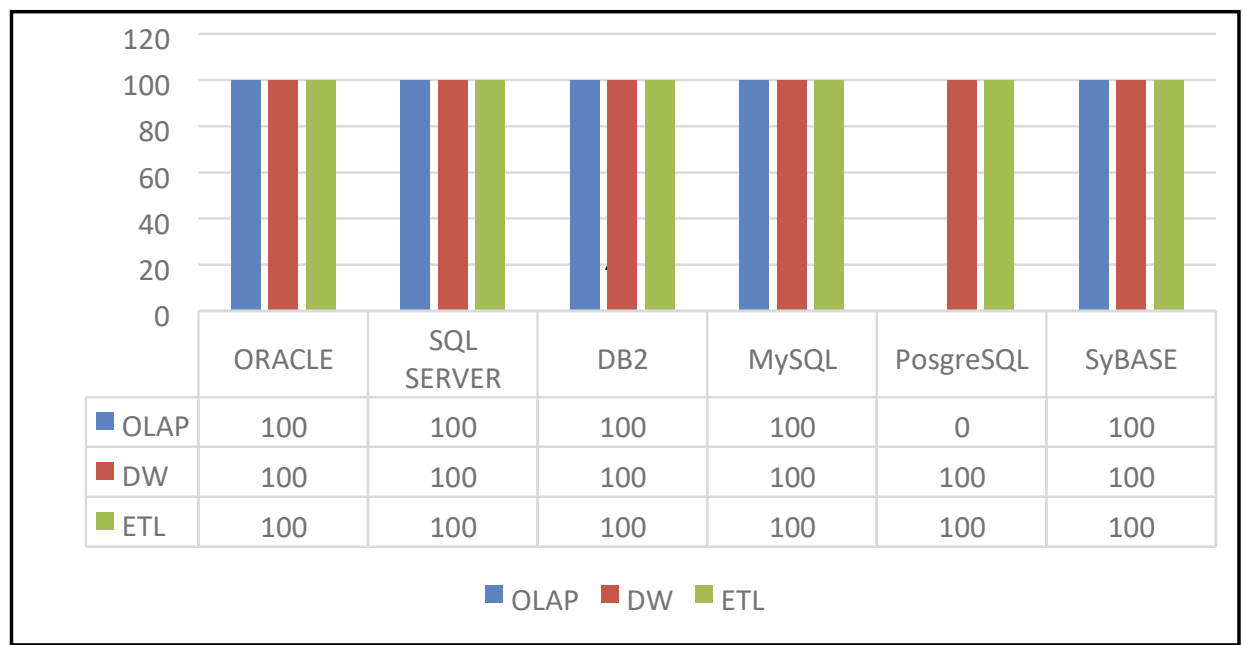

\section{(Fuente: Construcción propia a partir de manuales de referencia de los SGBD)}

La información anterior nos permite concluir que de todas las opciones evaluadas el único SGBD que no está diseñado para dar soporte de manera nativa a la inteligencia de negocios es PosgreSQL.

Ahora bien, para realizar un análisis con mayor profundidad se retomará el hecho que un solo SGBD puede contar con múltiples versiones, así haremos una comparativa por versión. La tabla 5 resume las versiones por SGBD obtenidas todas a partir del sitio web del proveedor.

\section{TABLA No. 5. Versiones por SGBD}

\begin{tabular}{|l|l|}
\hline SGBD & Extracción \\
\hline ORACLE & $\begin{array}{l}\text { Oracle Database Enterprise Edition } \\
\text { Oracle Database Standard Edition } \\
\text { Oracle Database Standard Edition One } \\
\text { Oracle Database Personal Edition } \\
\text { Oracle Cloud File System } \\
\text { Oracle Database Mobile Server } \\
\text { Oracle Database Media Pack }\end{array}$ \\
\hline SQL & $\begin{array}{l}\text { SQL Enterprise Edition } \\
\text { SERVER }\end{array}$ \\
& $\begin{array}{l}\text { SQL Business Intelligence } \\
\text { SQL Express Edition }\end{array}$ \\
\hline DB2 & $\begin{array}{l}\text { DB2 Connect Application Server Edition } \\
\text { DB2 Connect Enterprise Edition } \\
\text { DB2 Connect Personal Edition } \\
\text { DB2 Advanced Enterprise Server Edition } \\
\text { DB2 Developer Edition } \\
\text { DB2 Express Edition } \\
\text { DB2 Workgroup Server Edition }\end{array}$ \\
\hline
\end{tabular}




\begin{tabular}{|l|l|}
\hline MySQL & $\begin{array}{l}\text { MySQL Cluster Carrier Grade Edition } \\
\text { MySQL Enterprise Edition } \\
\text { MySQL Standard Edition } \\
\text { MySQL Community Edition }\end{array}$ \\
\hline PosgreSQL & PosgreSQL official core \\
\hline SYBASE & $\begin{array}{l}\text { ASE Deveoloper edition } \\
\text { ASE enterprise edition } \\
\text { ASE small business edition }\end{array}$ \\
\hline
\end{tabular}

Fuente: Elaboración propia a partir de manuales de referencia de los SGBD.

PosgreSQL hasta el momento el SGBD que no ofrece funcionalidades nativas para inteligencia de negocio es también el único que posee únicamente una versión, llama la atención que aunque, exceptuando a PosgreSQL, todos ofrecen diferentes versiones, no todos ofercen una versión gratuita tal como se puede apreciar en la tabla 6.

TABLA No. 6. SGBD versiones gratuitas

\begin{tabular}{|l|c|}
\hline SGBD & Posee versión gratuita \\
\hline ORACLE & No \\
\hline SQL SERVER & No \\
\hline DB2 & Sí \\
\hline MySQL & Sí \\
\hline PosgreSQL & No \\
\hline SYBASE & . \\
\hline
\end{tabular}

Fuente: Elaboración propia a partir de manuales de referencia de los SGBD

\subsection{Soluciones Técnicas, principales características.}

Los SGBD que si poseen un soporte para inteligencia de negocios no lo facilitan en las versiones gratuitas ni en las más económicas, éstos forman parte de la opción más costosa de cada compañía. De éstos módulos entonces podemos enumerar las siguientes características:

1. Estas funciones para soporte de Inteligencia de Negocios se comercializan a parte de la versión estándar y de las versiones gratuitas de los SGBD.

2. Únicamente ORACLE lo ofrece en sus versiones comerciales superiores al estándar.

3. IBM facilita una SUITE denominada COGNOS como solución directa a la inteligencia de negocios.

Si bien las versiones gratuitas no facilitan de manera nativa una respuesta para inteligencia de negocios existen en el mercado un buen número de herramientas que, pegándose a estos SGBD, permiten obtener inteligencia de negocio. 


\subsection{Requerimientos Técnicos}

Otro factor que debe tomarse en cuenta para poder o no hacer uso de un SGBD es precisamente la demanda de recursos que necesite, para ello es necesario establecer los requerimientos técnicos que poseen estas herramientas, en la tabla 7 puede apreciarse a la par de cada SGBD los requerimientos de hardware que deben cumplirse.

TABLA No. 7. Requerimientos de hardware ${ }^{\star}$

\begin{tabular}{|c|c|}
\hline SGBD & Extracción \\
\hline ORACLE & $\begin{array}{ll}\text { - } & 6 \text { GB disco duro. } \\
\text { - } & 4 \text { GB DDR 2 RAM } \\
\text { - } & 2 \mathrm{GHZ} \text { o superior } \\
\text { - } & \text { Intel x86, AMD64 Intel EM64T }\end{array}$ \\
\hline $\begin{array}{l}\text { SQL } \\
\text { SERVER }\end{array}$ & $\begin{array}{l}\text { - } 6 \text { GB de disco duro, unidad de DVD } \\
\text { - } \quad 4 \text { GB DDR 2 RAM } \\
\text { - } 2 \text { GHZ o superior } \\
\text { - } \quad \text { Procesador x64: AMD Opteron, AMD Athlon 64, Intel Xeon compatible con Intel } \\
\text { EM64T Intel Pentium IV compatible con EM64T } \\
\text { - Procesador x86: compatible con Pentium III o superior }\end{array}$ \\
\hline DB2 & $\begin{array}{l}\text { - } 1 \text { GB de RAM. } \\
1 \text { GB de disco duro. }\end{array}$ \\
\hline MySQL & $\begin{array}{ll}\text { - } & 300 \mathrm{MB} \text { de disco duro } \\
\text { - } & 256 \mathrm{MB} \text { de RAM } \\
\text { - } & 600 \mathrm{MHz} \\
\text { - } & \text { Pentium III }\end{array}$ \\
\hline SYBASE & $\begin{array}{ll}\cdot & 127 \mathrm{MB} \text { RAM } \\
\cdot & 700 \mathrm{MB} \text { disco duro }\end{array}$ \\
\hline
\end{tabular}

Para versiones que cuentan con soporte nativo para inteligencia de negocio

Fuente: Elaboración propia a partir de manuales de referencia de los SGBD.

En cuanto a hardware podemos ver que ORACLE y SQL server son los SGBD que requieren un equipo especial para estas funciones, en la tabla 7.1 se muestra el precio de un hardware similar (con mejores especificaciones) a los requerimientos técnicos mínimos.

TABLA No. 7.1 Costos Por Requerimientos de hardware

\begin{tabular}{|l|c|}
\hline SGBD & USD \$ 2,545.62 \\
\hline ORACLE & USD \$ 2,545.62 \\
\hline $\begin{array}{l}\text { SQL } \\
\text { SERVER }\end{array}$ & USD \$ $1,558.15$ \\
\hline DB2 & USD \$ $1,027.99$ \\
\hline MySQL & USD \$ 996.07 \\
\hline SYBASE & \\
\hline
\end{tabular}

Fuente: Elaboración propia a partir de precios de la página web del fabricante, consultados el 22/febrero/2016. 
Por otro lado, TODOS los SGBD se ejecutan en los sistemas operativos de mayor uso lo único es que algunos requieren (para trabajar en equipos MS Windows) de un software adicional tal como se aprecia en la tabla 8.

TABLA No. 8. Requerimientos de software ${ }^{\star}$

\begin{tabular}{|c|c|}
\hline SGBD & tracción \\
\hline $\begin{array}{l}\text { SQL } \\
\text { SERVER }\end{array}$ & $\begin{array}{ll}\text { - } & \text { NET } 4.0 \text { es un requisito para SQL Server } 2012 . \\
\text { - } & \text { Windows PowerShell } 2.0 \\
\text { - } & \text { Internet Explorer } 7\end{array}$ \\
\hline DB2 & - Windows Service Pack 2 \\
\hline MySQL & $\begin{array}{l}\text { - Microsoft Windows Installer Version } 2.0 \text { o superior y Microsoft .Net Framework } \\
\text { Versión } 2.0\end{array}$ \\
\hline SYBASE & $\begin{array}{ll}\text { - } & \text { Microsoft Visual C++ } 2005 \text { Service Pack } 1 \text { Redistributable Package and KB973544 } \\
\text { - } & \text { Microsoft Visual Studio } 2005 \text { Service Pack } 1 \text { and KB971090 }\end{array}$ \\
\hline
\end{tabular}

Para versiones que cuentan con soporte nativo para inteligencia de negocio Fuente: Elaboración propia a partir de manuales de referencia de los SGBD.

\subsection{Costos por licenciamiento}

Mientras que en la tabla 8 se facilita suficiente información para poder determinar el costo del equipo donde se almacenará el SGBD en la tabla 9 se muestran los costos por versión tomando como referencia únicamente las 3 versiones más aptas para el soporte a inteligencia de negocio

TABLA No. 9. Costos Por Licenciamiento (2014)

\begin{tabular}{|l|l|l|l|}
\hline \multicolumn{2}{|c}{ Estandar } & Enterprise & BI \\
\hline DB2 & USD \$ 579.00 & USD \$ 24,900.00 & USD \$ 94,000.00 \\
\hline ORACLE & USD \$ 497.56 & USD \$ 51390.28 & \\
\hline SQL SERVER & USD \$ 1,793.00 & USD \$ 6,874.00 & USD \$ 8,592.00 \\
\hline SYBASE & USD \$ 1487.00 & USD \$ 39,115.00 & \\
\hline MySQL & USD \$ 2,000.00 & USD \$ 5,000.00 & USD \$ 10,000.00 \\
\hline
\end{tabular}

Fuente: Elaboración propia a partir de manuales de referencia de los SGBD

IBM es la empresa con el software para soporte a inteligencia de negocio más oneroso del mercado seguido por ORACLE y terminando con SYBASE.

Por otra parte, el SGBD más económico con soporte a inteligencia de negocio es MySQL.

En la tabla 9.1 podemos encontrar la inversión que se debe hacer en cuanto a capacitaciones certifica- 
das para cada uno de los SGBD para un mejor uso mediante a personal altamente capacitados, los precios incluyen los niveles de expertos en el dominio de las funciones nativas y otras herramientas (para los SGBD que no poseen dichas funciones) así como también técnicas para la inteligencia de negocio.

\section{TABLA No. 9.1. Inversiones}

\begin{tabular}{|l|l|}
\hline \multicolumn{1}{|c|}{ SGBD } & USD \$ $3,810.00$ \\
\hline DB2 & USD \$ 3,374.00 \\
\hline ORACLE & USD \$ 2,400.00 \\
\hline SQL SERVER & USD \$ 2,393.00 \\
\hline SYBASE & USD \$ 3,479.00 \\
\hline MySQL & \\
\hline
\end{tabular}

Fuente: Elaboración propia a partir de precios de las páginas web de los partners, consultados el 22/febrero/2016.

\subsection{Software libre vrs software comercial}

De los seis SGBD presentados el 50\% respondían a la categoría del software libre (ver tabla 10), los sistemas comerciales contaban todos con funciones nativas que facilitan su uso para inteligencia de negocios.

\section{TABLA No. 10. SGBD Libres y Comerciales}

\begin{tabular}{|l|c|c|}
\hline SGBD & & Posee elementos libres \\
\hline ORACLE & $\mathrm{X}$ & \\
\hline SQL & & $\mathrm{X}$ \\
\hline DB2 & & $\mathrm{X}$ \\
\hline MySQL & $\mathrm{X}$ & $\mathrm{X}$ \\
\hline PosgreSQL & $\mathrm{X}$ & \\
\hline SYBASE & & \\
\hline
\end{tabular}

Fuente: Elaboración propia a partir de manuales de referencia de los SGBD.

EL software libre no posee la misma variedad de productos que su homologo propietario sin embargo existen herramientas en el mercado que solventan estas situaciones. 


\section{CONCLUSIONES E IMPLICACIONES}

1. A partir de la muestra empleada podemos concluir que el $50 \%$ de los SGBD ofrecen una versión gratuita.

2. Aunque en el mercado existen software para apoyo a inteligencia de negocios lo más recomendable es hacer uso de un sistema de información cuyo SGBD posea ya las funciones nativas.

3. Los SGBD pertenecientes al software libre carecen de funcionalidades OLAP nativas sin embargo siempre es posible hacer uso de herramientas de terceros.

4. Hay que tener especial atención al momento de implementar un ETL para prevenir tomas de decisiones erróneas.

5. Es propicio indagar sobre qué tan eficientes son los sistemas de terceros que dotan de inteligencia de negocios a SGBD que carecen de estas funcionalidades.

6. Podría ser propicio indagar sobre que SGBD poseen las empresas de la región y de si lo utilizan como herramienta para le toma de decisiones.

7. De los sistemas propietarios SQL es el menos costoso tanto en certificación como en obtención de su licencia mientras que MySQL se presenta como el SGBD más barato de todos (libres o propietarios) pero su certificación si representa una inversión mayor a la de otros gestores.

8. En cuanto a inversión en hardware ORACLE es el SGBD más exigente y SYBASE el más generoso. 


\section{REFERENCIAS BIBLIOGRÁFICAS}

Alvarez, E., Gorza, L., \& Lesie, G. (5 de febrero de 2011). scribd . Obtenido de Apunte BI: http://es.scribd.com/ doc/48897874/16/Drill-Down-y-Roll-Up

Araníbar S., J. C. (2003). Inteligencia de negocios. Universidad Católica Boliviana, 95-101. Obtenido de http:// www.scielo.org.bo/pdf/rcc/n12/a10.pdf

Gascón, M. d., \& Castellar-Busó, V. (19 de abril de 2001). RED científica ciencia, Tecnología y Pensamiento. Obtenido de Cómo diseñar grandes variables en bases de datos multidimensionales: http://www.redcientifica.com/doc/doc200104190004.html

IBM. (5 de diciembre de 2013). IBM DB2. Obtenido de IBM DB2: http://www-

01.ibm.com/software/data/db2/

Llombart, Ó. A. (9 de septiembre de 2004). El diario exterior - DAA contenidos digitales.

Obtenido de BI: Inteligencia aplicada al negocio: http://www.eldiarioexterior.com/anterior/conocimiento/ docs/BI_Inteligencia_aplicada_al_negocio.pdf

Luhn, H. P. (1958). A Business Intelligence System. IBM Journal.

Macías Mesa, J. A., \& Artola Pimentel, M. d. (2009). POSIBILIDADES ESTRATÉGICAS QUE BRINDA LA INTELIGENCIA EMPRESARIAL. Alta dirección, 1-10. Obtenido de

http://www.bibliociencias.cu/gsdl/collect/eventos/index/assoc/HASH017d.dir/doc.pdf

Microsoft. (5 de diciembre de 2013). Microsoft SQL Server. Obtenido de Microsoft SQL Server: http://www. microsoft.com/es-es/sqlserver/default.aspx

ORACLE - MySQL. (5 de diciembre de 2013). ORACLE - MYSQL. Obtenido de MySQL:

http://www.mysql.com/

ORACLE. (5 de diciembre de 2013). ORACLE. Obtenido de ORACLE:

http://www.oracle.com/

Oramas, J. (2009). La inteligencia de negocios, un concepto informático. Revista Sistemas (111),

42-51. Recuperado el 5 de diciembre de 2013, de http://acishost.acis.org.co/fileadmin/Revista_111/uno.pdf

PostgreSQL. (5 de diciembre de 2013). PostgreSQL. Obtenido de PostgreSQL:

http://www.postgresql.org.es/

SYBASE. (5 de diciembre de 2013). SYBASE. Obtenido de SYBASE: http://www.sybase.com

Zambrano Ramírez, R. (2008). Sistemas Gestores de Bases de Datos. Revista Digital Innovación y Experien-

cias Educativas, 1-9. Obtenido de http://www.csi-

csif.es/andalucia/modules/mod_ense/revista/pdf/Numero_14/RAQUEL_ZAMBRANO_

2.pdf

\section{AUTORIZACIÓN Y RENUNCIA}

El siguiente texto deberá aparecer en la sección; "Los (a) autores facultan a CEAT para publicar el escrito en los procedimientos de la conferencia. CEAT o los editores no son responsables por el contenido y las implicaciones de lo que esta expresado en el escrito.” 